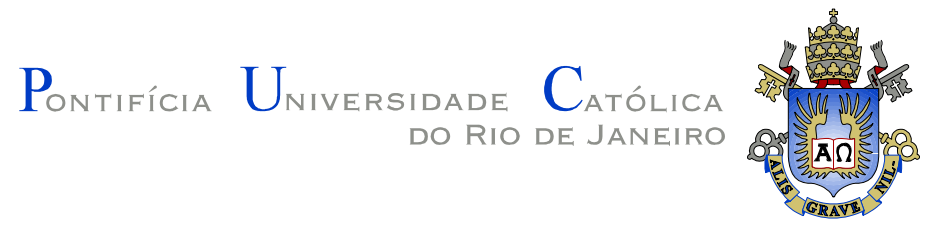

Rodrigo Bird Burgos

\title{
Análise de Estruturas Utilizando Wavelets de Daubechies e Interpolets de Deslauriers-Dubuc
}

Tese de Doutorado

Tese apresentada como requisito parcial para obtenção do título de Doutor pelo Programa de PósGraduação em Engenharia Civil da PUC-Rio.

Orientadores: Raul Rosas e Silva Marco Antonio Cetale Santos 
Rodrigo Bird Burgos

\title{
Análise de Estruturas Utilizando Wavelets de Daubechies e Interpolets de Deslauriers-Dubuc
}

Tese apresentada como requisito parcial para obtenção do título de Doutor pelo Programa de Pós-Graduação em Engenharia Civil da PUC-Rio. Aprovada pela Comissão Examinadora abaixo assinada.

\author{
Raul Rosas e Silva \\ Orientador \\ PUC-Rio \\ Marco Antonio Cetale Santos \\ Co-Orientador \\ UFF \\ Deane de Mesquita Roehl \\ PUC-Rio \\ Luiz Fernando Campos Ramos Martha \\ PUC-Rio \\ Paulo Batista Gonçalves \\ PUC-Rio \\ Luiz Eloy Vaz \\ UFRJ
}

Roque Luiz da Silva Pitangueira

UFMG

José Eugênio Leal

Coordenador(a) Setorial do Centro Técnico Científico - PUC-Rio

Rio de Janeiro, 18 de setembro de 2009 
Todos os direitos reservados. É proibida a reprodução total ou parcial do trabalho sem autorização da universidade, do autor e do orientador.

\section{Rodrigo Bird Burgos}

Graduou-se em Engenharia Civil pela PUC-Rio, em dezembro de 2002. Em fevereiro de 2005 defendeu sua Dissertação de Mestrado. Ingressou no doutorado em março de 2005.

Ficha Catalográfica

Burgos, Rodrigo Bird

Análise de estruturas utilizando wavelets de Daubechies e interpolets de Deslauriers-Dubuc / Rodrigo Bird Burgos ; orientadores: Raul Rosas e Silva, Marco Antonio Cetale Santos. - 2009.

185 f. : il. (color.) ; $30 \mathrm{~cm}$

Tese (Doutorado em Engenharia Civil)-Pontifícia Universidade Católica do Rio de Janeiro, Rio de Janeiro, 2009.

Inclui bibliografia

1. Engenharia civil - Teses. 2. Wavelets. 3. Interpolets. 4. MEF 5. Método de Wavelet-Galerkin. 6. Daubechies. 7. Flambagem. 8. Carga crítica. 9. Estabilidade estrutural. 10. Dinâmica estrutural. I. Silva, Raul Rosas e. II. Santos, Marco Antonio Cetale. III. Pontifícia Universidade Católica do Rio de Janeiro. Departamento de Engenharia Civil. IV. Título. 


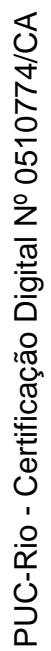

À minha filha Irene. 


\section{Agradecimentos}

Ao professor Raul Rosas e Silva pela amizade, dedicação, paciência e ensinamentos, além da confiança que demonstrou ao me propor este desafio.

Ao professor Marco Antonio Cetale Santos, principalmente por aceitar o desafio de me co-orientar.

Aos professores Luiz Fernando Martha e Paulo Batista Gonçalves pela ajuda fornecendo exemplos pela motivação.

Aos demais professores integrantes da banca examinadora.

À secretária Rita, pela inesgotável paciência, e aos demais integrantes do Departamento de Engenharia Civil da PUC-Rio.

À minha filha Irene, pela inspiração.

Aos meus pais, por terem me ajudado a me tornar quem sou.

À minha namorada Aline, pela ajuda com as figuras e pelo apoio.

Ao CNPQ e à FAPERJ pelo auxílio financeiro. 


\section{Resumo}

Burgos, Rodrigo Bird. Análise de Estruturas Utilizando Wavelets de Daubechies e Interpolets de Deslauriers-Dubuc. Rio de Janeiro, 2009. 185p. Tese de Doutorado - Departamento de Engenharia Civil, Pontifícia Universidade Católica do Rio de Janeiro.

Funções Wavelet de suporte compacto têm sido recentemente aplicadas na resolução numérica de equações diferenciais com resultados bastante promissores. A partir do sucesso do uso das wavelets de Daubechies em diversos métodos como o de Galerkin, surgiram novas famílias de wavelets para a resolução de problemas específicos. Nesse contexto, vale destacar uma família de wavelets com características de funções interpoladoras chamadas Interpolets. Este trabalho tem como uma de suas contribuições a formulação de elementos finitos baseados em funções wavelet de Daubechies e interpolets de Deslauriers-Dubuc para sua utilização em problemas dinâmicos como a propagação de ondas em estruturas, além de problemas não-lineares como o cálculo de cargas críticas de flambagem para colunas e pórticos. A partir dessa formulação, o Método de Wavelet-Galerkin foi adaptado para a solução direta das equações diferenciais através de uma implementação que não depende da discretização do sistema em graus de liberdade (formulação sem-malha ou meshless). Este tipo de abordagem permite também explorar ao máximo as propriedades de multirresolução das wavelets. Diversos exemplos com descontinuidades e não-linearidades foram estudados com êxito.

\section{Palavras-chave}

Wavelets; Interpolets; MEF; Método de Wavelet-Galerkin; Daubechies; Flambagem; Carga Crítica; Estabilidade Estrutural; Dinâmica Estrutural 


\section{Abstract}

Burgos, Rodrigo Bird. Structural Analysis with use of Daubechies Wavelets and Deslauriers-Dubuc Interpolets. Rio de Janeiro, 2009. 185p. Tese de Doutorado - Departamento de Engenharia Civil, Pontifícia Universidade Católica do Rio de Janeiro.

The use of compactly supported wavelet functions has become increasingly popular in the development of numerical solutions for differential equations. Daubechies wavelets have been successfully used as base functions in several schemes like the Galerkin Method. Meanwhile, Deslauriers and Dubuc developed a new wavelet family with interpolating characteristics called Interpolets. One important contribution presented in this work is the formulation of Finite Elements based in Daubechies wavelets and Deslauriers-Dubuc interpolets. Dynamic problems like wave propagation and structural stability problems were used as examples for the validation of the Finite Elements. The Wavelet-Galerkin Method has then been adapted for the direct solution of differential equations in a meshless formulation. This approach enables the use of a multiresolution analysis. Several examples with discontinuities and nonlinearities were studied successfully.

\section{Keywords}

Wavelets; Interpolets; FEM; Wavelet-Galerkin Method; Daubechies; Buckling; Critical Loads; Structural Stability; Structural Dynamics 


\section{Sumário}

1 Introdução 20

1.1. Organização do texto 26

2 Fundamentação Teórica 28

2.1. Método dos Elementos Finitos (MEF) 28

2.2. Método de Galerkin 29

2.3. Introdução às Wavelets 30

2.3.1. Propriedades das Wavelets 33

2.4. Análise Multirresolução 36

2.4.1. Aproximação de funções 39

2.4.2. Propriedades da Análise Multirresolução 40

2.4.2.1. Localização $\quad 40$

2.4.2.2. Momentos Nulos e Aproximação de Polinômios 41

2.4.2.3. Caracterização de Regularidade e Representação de $\begin{array}{ll}\text { Singularidades } & 42\end{array}$

2.4.3. Exemplo de uma Análise Multirresolução 42

2.5. Tipos de Wavelets 46

2.5.1. Wavelets Ortonormais $\quad 47$

2.5.1.1. Wavelets de Daubechies $\quad 48$

2.5.1.2. Wavelets de Daubechies Bidimensionais 49

2.5.2. Interpolets de Deslauriers-Dubuc 50

2.6. Método de Wavelet-Galerkin 52

2.6.1. Avaliação correta dos coeficientes de conexão 54

2.6.2. Incorporação das Condições de Contorno 56

2.7. Construção das Wavelets de Daubechies $\quad 57$

2.7.1. Determinação dos coeficientes de filtro 58

2.7.1.1. Exemplo de cálculo dos coeficientes da wavelet DB4 59

2.7.2. Obtenção da função de escala 60

2.7.2.1. Exemplo de cálculo dos valores da wavelet DB4 62 
2.7.3. Integrais das Wavelets de Daubechies 64

2.7.3.1. Exemplo de cálculo de integral Daubechies DB4 66

2.7.3.2. Produto interno com polinômios 67

2.7.4. Derivadas das Wavelets de Daubechies 69

2.7.5. Coeficientes de Conexão das Wavelets de Daubechies $\quad 71$

2.7.5.1. Coeficientes de Conexão Impróprios 71

2.7.5.2. Coeficientes de Conexão para Matrizes de Rigidez, Massa e Geométrica $\quad 75$

2.7.5.3. Coeficientes de Conexão para Carregamento 78

2.7.5.4. Coeficientes de Conexão de Três Funções de Escala 79

2.7.5.5. Coeficientes de Conexão de Polinômios e Duas Funções de Escala $\quad 80$

2.7.5.6. Cálculo dos Momentos 82

2.8. Construção das Interpolets $\quad 84$

2.8.1. Integrais das Interpolets 86

2.8.2. Derivadas e Coeficientes de Conexão das Interpolets 87

3 Aplicação do Método de Wavelet-Galerkin 89

3.1. Equação Diferencial Harmônica 89

3.1.1. Solução pelo Método das Condições de Contorno Fictícias $\quad 90$

3.1.2. Solução Utilizando Coeficientes de Conexão Próprios 94

3.1.2.1. Solução em Níveis Seguintes 95

3.2. Equação Diferencial de uma Barra de Treliça 97

3.3. Equação Diferencial de uma Viga à Flexão 98

3.4. Viga Submetida a uma Carga Axial 100

3.5. Viga Sobre Base Elástica 101

4 Formulação dos Elementos Unidimensionais 103

4.1. Elemento de Treliça 103

4.1.1. Funções de Forma Wavelet de Treliça 106

4.1.2. Matrizes de Rigidez e de Massa do Elemento de Treliça 108

4.2. Elemento de Viga 109

4.2.1. Funções de Forma Wavelet de Viga 111 
4.2.2. Matrizes de Rigidez, Geométrica e de Massa do Elemento de Viga

4.3. Cargas Equivalentes Nodais 115

$\begin{array}{ll}\text { 4.4. Wavelets de Alta Ordem } & 116\end{array}$

5 Validação dos Elementos 117

5.1. Análise Estática 117

5.1.1. Coluna sob Ação do Peso Próprio 117

5.1.2. Viga sob Ação do Peso Próprio 119

5.1.3. Viga Biengastada Submetida a Carregamento Linear 121

5.1.4. Viga sobre Base Elástica Submetida a Carregamento

$\begin{array}{ll}\text { Concentrado } & 124\end{array}$

5.1.5. Casca Cilíndrica Axissimétrica 127

5.2. Análise Dinâmica 130

5.2.1. Cálculo de Freqüências Naturais de Vibração 130

5.2.2. Propagação de Ondas 131

5.3. Análise de Instabilidade 135

5.3.1. Obtenção de Cargas Críticas e Modos de Flambagem de Colunas Clássicas 135

5.3.2. Cálculo de Cargas Críticas do Pórtico de Roorda 136

$\begin{array}{ll}\text { 5.4. Discussão dos Resultados } & 137\end{array}$

6 Método de Wavelet-Galerkin Modificado 138

6.1. Analogias entre o Método de Wavelet-Galerkin Modificado

$\begin{array}{ll}\text { e o MEF } & 139\end{array}$

6.2. Imposição das Condições de Contorno no Espaço das Funções 140

6.3. Demonstração da Equivalência dos Métodos 143

6.4. Imposição de Cargas e Momentos Concentrados 145

7 Exemplos do Método Proposto 146

7.1. Viga Biapoiada com Carga Concentrada 146

7.2. Coluna com Área Variável 148

7.3. Viga com Altura Variável 153

7.4. Cálculo de Cargas Críticas de Peso Próprio de Colunas 158 
7.5. Cálculo de Cargas Críticas de Vigas sobre Base Elástica

8 Conclusões e Propostas para Trabalhos Futuros

9 Referências Bibliográficas

Apêndice A Implementação Computacional

Apêndice B Aplicação a Placas Finas

180

Apêndice C Exemplo de Montagem da Matriz dos Coeficientes de Conexão 


\section{Lista de símbolos}

\section{Romanos}

A - área da seção transversal

$a_{i}-$ coeficientes de filtro da função de escala

c - rigidez da base elástica

$c_{i}^{j}$ - coeficientes de aproximação da função de escala

$d_{i}-$ coeficientes de interpolação

$d_{i}^{j}$ - coeficientes de aproximação da função wavelet

E - módulo de elasticidade

f - vetor de forças nodais

I - momento de inércia da seção reta

$\mathbf{K}$ - matriz de rigidez elástica

$\mathbf{k}$ - matriz de rigidez elástica do elemento

G - matriz geométrica

g - matriz geométrica do elemento

$h_{i}-$ coeficientes de filtro da função wavelet

j, m - nível de resolução

$\mathrm{L}$ - comprimento do elemento ou estrutura

$L_{i}^{j}$ - momento j da função wavelet com translação i

m - matriz de massa do elemento

M - matriz de massa da estrutura

$M_{i}^{j}$ - momento j da função de escala com translação $i$

$\mathrm{N}_{\mathrm{i}}(\mathrm{x})$ - funções de forma de elementos finitos

$\mathrm{N}$ - ordem da função wavelet

$P$ - força axial

$q(x)$ - função de carregamento transversal

$\mathbf{R}$ - vetor das reações de apoio

$\mathrm{u}$ - deslocamento axial

V - subespaço de resolução da função de escala 
w -deslocamento transversal

W - subespaço de resolução da função wavelet

\section{Gregos}

$\Pi$ - energia potencial total

$\Delta \Pi$ - variação da energia potencial total

$\delta$ - delta de Dirac

$\mu$ - parâmetro de carga

$\sigma-$ tensão axial

$\varepsilon$ - deformação axial

$\Delta$ - variação

$\omega$ - freqüência de vibração

$\rho$ - massa por unidade de volume

$\gamma$ - peso por unidade de volume

$\theta$ - grau de liberdade de rotação

$\varphi$ - função de escala

$\psi$ - função wavelet

$\Theta$ - malha diádica

$\Gamma$ - coeficiente de conexão próprio

$\Lambda$ - coeficiente de conexão impróprio

$\Phi$ - vetor das componentes da função de escala

$\xi$ - coordenada espacial adimensional

\section{Outros}

$\mathcal{L}^{2}-$ espaço das funções quadrado integráveis

$\mathcal{P}$ - projeção no espaço das funções de escala

$\mathcal{Q}$ - projeção no espaço das funções wavelet 


\section{Lista de figuras}

Figura 1 - Descontinuidades num modelo estrutural

Figura 2 - Decomposição de uma função contendo descontinuidades em séries de Fourier e wavelets

Figura 3 - Comparação entre uma função senoidal e uma função wavelet

Figura 4 - Decomposição de um sinal (função) em componentes senoidais

Figura 5 - Decomposição de uma função qualquer em wavelets de diferentes escalas e posições

Figura 6 - Translações da wavelet DB4 que contém o intervalo [0,1] 35

Figura 7 - Translações ponderadas da função de escala e somatório que resulta na reta $y=x$ no intervalo $[0,1]$

Figura 8 - Os subespaços e suas funções associadas

Figura 9 - Subespaços, funções de escala e wavelet

Figura 10 - Funções de escala e wavelet de Daubechies de ordem $N=4$

Figura 11 - Aproximação de uma parábola por wavelets em diferentes níveis

Figura 12 - Wavelets de Daubechies

Figura 13 - Função de escala bidimensional de Daubechies de ordem $\mathrm{N}=4$

Figura 14 - Representação física de uma malha diádica

Figura 15 - Diferentes iterações para a obtenção da função de escala DB4

Figura 16 - Interpolet de Deslauriers-Dubuc de ordem N = 4

Figura 17 - Oscilador harmônico $\quad 89$

Figura 18 - Solução da equação harmônica por DB6 e DB12 93

Figura 19 - Aproximação da solução da equação harmônica com 
Figura 20 - Funções de forma de treliça baseadas na wavelet DB4 106

Figura 21 - Três primeiras funções de forma de treliça baseadas na interpolet IN4

Figura 22 - Três últimas funções de forma de treliça baseadas na interpolet IN4 107

Figura 23 - Graus de liberdade de um elemento de viga DB8

Figura 24 - Duas primeiras funções de forma de deslocamento do elemento de viga baseado na wavelet DB8

Figura 25 - Função de forma de rotação unitária na extremidade direita do elemento de viga baseado na wavelet DB8

Figura 26 - Funções de forma de deslocamento do elemento de viga baseado na interpolet IN4

Figura 27 - Funções de forma de rotação do elemento de viga baseado na interpolet IN4

Figura 28 - Esquema estrutural de uma coluna sob ação de seu peso próprio

Figura 29 - Resultados para o deslocamento da coluna sob peso próprio

Figura 30 - Esquema estrutural de uma viga biapoiada sob ação de seu peso próprio

Figura 31 - Deslocamentos para a viga biapoiada sob peso próprio

Figura 32 - Momento fletor obtido em alguns pontos por interpolação 120

Figura 33 - Modelo estrutural da viga submetida a carregamento linear em metade de seu vão

Figura 34 - Deslocamento para os elementos DB10 e DB12 122

Figura 35 - Momento fletor para os elementos DB10 e DB12 122

Figura 36 - Deslocamento para os elementos IN4 e IN6 123

Figura 37 - Momento fletor para os elementos IN4 e IN6 123

Figura 38 - Viga sobre base elástica submetida a carga concentrada 124

Figura 39 - Deslocamento para a viga sobre base elástica utilizando elementos DB10 e DB12

Figura 40 - Momento fletor para a viga sobre base elástica utilizando elementos DB10 e DB12 
Figura 41 - Deslocamento para a viga sobre base elástica utilizando elementos IN6 e IN8

Figura 42 - Momento fletor para a viga sobre base elástica utilizando elementos IN6 e IN8

Figura 43 - Modelagem da casca cilíndrica axissimétrica como uma viga sobre base elástica

Figura 44 - Deslocamento para a casca cilíndrica axissimétrica modelada com elementos DB10 e DB12

Figura 45 - Momento fletor para a casca cilíndrica axissimétrica modelada com elementos DB10 e DB12

Figura 46 - Deslocamento para a casca cilíndrica axissimétrica modelada com elementos IN6 e IN8

Figura 47 - Momento fletor para a casca cilíndrica axissimétrica modelada com elementos IN6 e IN8

Figura 48 - Barra submetida a uma onda de deslocamento axial $u(t)$

Figura 49 - Deslocamento aplicado à extremidade da barra

Figura 50 - Espectro do deslocamento aplicado

Figura 51 - Detalhe do deslocamento do ponto médio da barra obtido utilizando elementos Interpolet e elementos de treliça padrão

Figura 52 - Estados do deslocamento em todas as posições e tempos utilizando elementos de treliça padrão e elementos IN4

Figura 53 - Pórtico de Roorda com dois tipos de carregamento

Figura 54 - Montagem da matriz de coeficientes de conexão no intervalo $\left[0,2^{\mathrm{m}}\right]$

Figura 55 - Modelo estrutural de uma viga com carga concentrada 146

Figura 56 - Diagramas para DB10 em nível 8 147

Figura 57 - Diagramas para DB10 em nível 10 147

Figura 58 - Diagramas para IN4 em nível 6 147

Figura 59 - Diagramas para IN4 em nível 8

Figura 60 - Coluna com área variável submetida a carga concentrada 149

Figura 61 - Tensão na coluna com área variável 152

Figura 62 - Detalhe da tensão na coluna com área variável na região de maior gradiente 
Figura 63 - Viga com altura variável (mísula)

Figura 64 - Carregamento concentrado para a viga com mísula 156

Figura 65 - Carregamento distribuído na viga com mísula 156

Figura 66 - Erro relativo no momento fletor do engaste em função dos graus de liberdade utilizados por cada método

Figura 67 - Equilíbrio de momentos de uma coluna submetida ao peso próprio no estado pós-flambagem 158

Figura 68 - Esforço normal da coluna sujeita ao peso próprio 159

Figura 69 - Erro relativo na carga crítica de peso próprio em função dos graus de liberdade utilizados por cada método

Figura 70 - Diferentes condições de contorno para as quais vale a mesma expressão do esforço normal

Figura 71 - Viga biapoiada sobre base elástica submetida a carga axial

Figura 72 - Dois primeiros modos de flambagem da viga biapoiada sobre base elástica

Figura 73 - Modos de flambagem para a mesma carga crítica da viga biapoiada sobre base elástica

Figura 74 - Primeiro modo de flambagem da viga biapoiada sobre base elástica infinitamente longa

Figura 75 - Viga infinitamente longa sobre base elástica livre nas extremidades

Figura 76 - Modos de flambagem simétrico e antissimétrico da viga infinitamente longa sobre base elástica livre nas extremidades 


\section{Lista de tabelas}

Tabela 1 - Coeficientes de filtro das interpolets de Deslauriers-Dubuc 85

Tabela 2 - Momento fletor no ponto de aplicação da carga 126

Tabela 3 - Momento fletor na base da casca 130

Tabela 4 - Freqüências de vibração 131

Tabela 5 - Resultados para o cálculo da carga crítica de flambagem comparados com os obtidos por 3 elementos de viga padrão

Tabela 6 - Resultados para o cálculo da carga crítica de flambagem do pórtico de Roorda comparados com os obtidos por 3 elementos de viga padrão 136

Tabela 7 - Tensões e deslocamento para $\alpha=1 / 2$. 151

Tabela 8 - Tensões e deslocamento para $\alpha=1 / 10$. 152

Tabela 9 - Tensões e deslocamento para $\alpha=1 / 100$. 152

Tabela 10 - Momentos fletores adimensionais para a viga com mísula e carregamento concentrado $\left(\times 10^{-2}\right)$

Tabela 11 - Momentos fletores adimensionais para a viga com mísula e carregamento distribuído $\left(\times 10^{-2}\right)$

Tabela 12 - Resultados obtidos para cargas críticas de peso próprio da coluna engastada e livre

Tabela 13 - Cargas críticas de peso próprio para as condições de contorno: biapoiada, biengastada, engastada e apoiada e apoiada e engastada

Tabela 14 - Resultados para as duas primeiras cargas críticas de uma viga biapoiada sobre base elástica

Tabela 15 - Resultados para as duas primeiras cargas críticas de uma viga biapoiada sobre base elástica com $c=4 \pi^{4} \mathrm{EI} / \mathrm{L}^{4}$

Tabela 16 - Resultados de cargas críticas para uma viga biapoiada sobre base elástica infinitamente longa

Tabela 17 - Resultados de cargas críticas para uma viga infinitamente longa sobre base elástica livre nas extremidades 
Tabela 18 - Resultados para cargas críticas de viga sobre base elástica com as condições de contorno: engastada e livre, biengastada e engastada e apoiada 